\title{
Simultaneous analysis of T helper subsets (Th1, Th2, Th9, Th17, Th22, Tfh, Tr1 and Tregs) markers expression in periapical lesions reveals multiple cytokine clusters accountable for lesions activity and inactivity status
} \author{
SILVA ${ }^{4}$, Gustavo Pompermaier GARLET ${ }^{1}$

\footnotetext{
1- Department of Biological Sciences, Bauru School of Dentistry, University of São Paulo (FOB/USP), Bauru, SP, Brazil.

2- Department of Conservative Dentistry, Faculty of Dentistry, University of Chile (UChile), Santiago, Chile.

3- Department of Dental Sciences, University of Cuiaba (FOC-UNIC), Cuiaba, MT, Brazil.

4- Department of Endodontics, School of Dentistry, University of Texas Health Science Center at Houston, Houston, USA.

5- Department of Biological and Allied Health Sciences, Sacred Heart University (USC), Bauru, SP, Brazil.

6- Department of Periodontology, Dental Research Division, Guarulhos University (UNG), Guarulhos, SP, Brazil.
}

Ana Claudia ARAUJO-PIRES ${ }^{1}$, Carolina Favaro FRANCISCONI' , Claudia Cristina BIGUETTI' Franco CAVALLA $^{1,2}$, Andreza Maria Fabio ARANHA ${ }^{1,3}$, Ariadne LETRA ${ }^{4}$, Ana Paula Favaro TROMBONE ${ }^{5}$, Marcelo FAVERI ${ }^{6}$, Renato Menezes

Corresponding address: Gustavo Pompermaier Garlet - Departamento de Ciências Biológicas - Faculdade de Odontologia de Bauru (FOB/USP) - Al. Octávio Pinheiro Brisola, 9-75 - 17012-901 - Bauru - SP - Brazil - Phone: +55 (14) 3235-8274 - Fax: +55 (14) 3223-4679 - E-mail: garletgp@usp.br

Submitted: March 28, 2014 - Modification: May 16, 2014 - Accepted: May 20, 2014

\section{ABSTRACT}

\begin{abstract}
Drevious studies demonstrate that the balance between pro- and anti-inflammatory mediators determines the stable or progressive nature of periapical granulomas by modulating the balance of the osteoclastogenic factor RANKL and its antagonist OPG. However, the cytokine networks operating in the development of periapical lesions are quite more complex than what the simple pro- versus anti-inflammatory mediators' paradigm suggests. Here we simultaneously investigated the patterns of Th1, Th2, Th9, Th17, Th22, $\operatorname{Thf}, \operatorname{Tr} 1$ and Tregs cytokines/markers expression in human periapical granulomas. Methods: The expression of TNF- $\alpha$, IFN- $\gamma$, IL-17A, IL23, IL21, IL-33, IL-10, IL-4, IL-9, IL-22, FOXp3 markers (via RealTimePCR array) was accessed in active/progressive $(\mathrm{N}=40)$ versus inactive/stable $(\mathrm{N}=70)$ periapical granulomas (as determined by RANKL/OPG expression ratio), and also to compare these samples with a panel of control specimens $(N=26)$. A cluster analysis of 13 cytokine levels was performed to examine possible clustering between the cytokines in a total of 110 granulomas. Results: The expression of all target cytokines was higher in the granulomas than in control samples. TNF- $\alpha$, IFN- $\gamma$, IL-17A and IL-21 mRNA levels were significantly higher in active granulomas, while in inactive lesions the expression levels of IL-4, IL-9, IL-10, IL-22 and FOXp3 were higher than in active granulomas. Five clusters were identified in inactive lesion groups, being the variance in the expression levels of IL-17, IL-10, FOXp3, IFN- $\gamma$, IL-9, IL-33 and IL-4 statistically significant (KW p<0.05). Three clusters were identified in active lesions, being the variance in the expression levels of IL-22, IL-10, IFN- $\gamma$, IL-17, IL-33, FOXp3, IL-21 and RANKL statistically significant (KW $\mathrm{p}<0.05)$. Conclusion: There is a clear dichotomy in the profile of cytokine expression in inactive and active periapical lesions. While the widespread cytokine expression seems to be a feature of chronic lesions, hierarchical cluster analysis demonstrates the association of TNF- $\alpha$, IL-21, IL-17 and IFN- $\gamma$ with lesions activity, and the association of FOXP3, IL-10, IL-9, IL-4 and IL-22 with lesions inactivity.
\end{abstract}

Keywords: Apical granuloma. Cytokine networks. Immunoregulation. Immunology. 


\section{INTRODUCTION}

Periapical lesions triggered by bacterial infection of pulpal and endodontic environment are characterized by the destruction of mineralized tissues surrounding the root apex as a consequence of the local host response ${ }^{19}$. In this context, cytokines play a major role in the modulation of inflammatory immune responses within the periapical microenvironment, and, therefore, are critical determinants of lesions outcome ${ }^{12,19}$. Previous studies demonstrate that the balance between pro- and anti-inflammatory mediators determines the stable or progressive nature of periapical granulomas by modulating the balance of the osteoclastogenic factor RANKL and its antagonist OPG ${ }^{12,29}$. However, the cytokine networks operating in the development of periapical lesions are quite more complex than what the relatively simple pro- versus anti-inflammatory mediators' paradigm could suggest ${ }^{5}$, being the pathogenesis of chronic inflammatory diseases influenced by several other cytokine classes ${ }^{12,19}$.

In this scenario, Th1 cytokines (IFN- $\gamma$, IL-12) have been associated with bone destruction and lesion progression, while its classic Th2 antagonists (IL-4, IL-10, and the recently described IL-33) are described to limit or attenuate the tissue damage ${ }^{19}$. Beyond the Th1/Th2 archetype, Th17 cells emerged as a $T$ subset with inflammatory properties involved in a series of infectious, autoimmune and osteolytic processes ${ }^{41}$. While the prototypical Th17 cytokine is IL-17, Th17 cells can also produce other effector cytokines with osteoclastogenic properties, such as IL-6 and IL-23, reinforcing the potential destructive role of Th17 subset in periapical lesions ${ }^{41}$. On the other hand, regulatory T cells (Tregs, a FOXp3+CD4+ subset) and Tr1 cells present suppressive effects on inflammatory osteolysis, thought to be mediated by cytokines such as TGF- $\beta$ and $\mathrm{IL}-10^{3,15,17}$. Interestingly, the Th17/Tregs archetype was suggested to influence the outcome of periapical lesions ${ }^{9,28}$.

Adding more complexity to the cytokine network in periapical lesions, Th9 and Th22 cytokines are expressed in human and experimental periapical lesions, where they are supposed to contribute to lesion stability ${ }^{1}$. IL-9 (the main Th9 product) and IL-22 (the Th22 signature cytokine) have been described as pleiotropic cytokines, whose pro- or anti-inflammatory activities may significantly differ depending on the overall cytokine milieu ${ }^{5}$. Other pleiotropic cytokines, such as IL-21 (a product of Th17 or T follicular helper [Tfh] cells), can also impact the overall immunoregulatory milieu, and also the osteoclastogenesis and bone resorption processes $^{25}$.

While previous studies describe the possible involvement of the mentioned cytokines in periapical lesions as a general rule, such mediators' expression have been investigated independently or in small sets $6,19,28$, which does not provide a reasonable understanding of the whole cytokine network in periapical environment. Indeed, considering the notable interplay between the cytokines ${ }^{5}$, only the simultaneous analysis of a broad cytokine panel can provide a picture of the overall immunoregulatory scenario operating at periapical lesions. Therefore, here we simultaneously investigated the patterns of Th1, Th2, Th9, Th17, Th22, Thf, Tr1 and Tregs cytokines/markers expression in human chronic periapical granulomas and their possible correlations with lesions activity pattern.

\section{MATERIAL AND METHODS}

\section{Samples}

This study had institutional review board approval of Bauru School of Dentistry, University of São Paulo. Patients presenting periapical lesions were referred to endodontic surgery after conventional root canal treatment failure; periapical lesions diagnosis was performed as previously described 29,30 , based on histopathological and radiographic analysis, being periapical lesions characterized radiographically as rarefaction lesions with the disappearance of the periodontal ligament space and discontinuity of the lamina dura. Treatment failure was defined as the presence of periradicular radiolucency that did not resolve, persisting as before acceptable endodontic treatment (i.e. having all canals instrumented and obturated, with no voids in the obturation mass, the apical terminus of the obturation at $1 / 1.5 \mathrm{~mm}$ from the radiographic apex), or that increased in size with evidences of continuous bone resorption ${ }^{2}$. Periapical granulomas $(\mathrm{N}=110)$ were collected from patients ( $N=110$, aged 19-59; 51 females and 59 males) during periapical surgery and divided in two roughly similar fragments and stored in both formalin (for routine histological examination; was performed after hematoxylin-eosin staining) and RNAlater (Ambion, Austin, TX) (for molecular analysis) solutions. Test samples were limited to granulomas, histopathologically defined by the presence of capillaries, inflammatory cells, macrophages, and without the presence of an epithelial lining. Periapical cysts, where cavities were further developed and lined by stratified squamous epithelium, and partially epithelized lesions (epithelized granulomas) were excluded from the study. Patients with medical conditions which need the use of systemic modifiers of bone metabolism or other assisted drug therapy (i.e. systemic antibiotics, anti-inflammatory medicines, hormonal therapy) during the last 6 months before the study were excluded. Patients with preexisting 
conditions, such as periodontal disease, and pregnant or lactating women were also excluded. Healthy periodontal ligament tissue samples $(N=26)$ obtained from premolars extracted for orthodontic purposes (patients aged 19-24 years, 12 females and 14 males) and stored in RNA later were used as control specimens. Lesions were also categorized into putative active (A) and inactive (I), based in the molecular profile of RANKL/OPG mRNA expression, as previously described ${ }^{29}$.

\section{RNA extraction and RealTime-PCR reactions}

Samples were submitted to molecular analyses as previously described ${ }^{30}$. In brief, total RNA was extracted from samples by using the RNeasy kit (Qiagen Inc, Valencia, CA) according to the manufacturers' instructions. The integrity of RNA samples was checked by analyzing $1 \mu \mathrm{g}$ of total RNA on 2100 Bioanalyzer (Agilent Technologies, Santa Clara, CA) according to the manufacturers' instructions. After RNA extraction, complementary DNA was synthesized by using $3 \mu \mathrm{g}$ of RNA through a reverse transcription reaction using QuantiTectRT kit (Qiagen Inc, Valencia, CA). All cytokines/Th markers (TNF- $\alpha$, IFN- $\gamma$, IL-17A, IL-23, IL-21, IL33, IL-10, IL-4, IL-9, IL-22, FOXp3) mRNA levels were measured by means of RealTimePCR using TaqMan chemistry (Invitrogen, Carlsbad, CA) in a Viia7 instrument (LifeTechnologies, Carlsbad, CA) using inventoried optimized primers/probes sets (Invitrogen, Carlsbad, CA), with basic reaction conditions $(40 \mathrm{cycles})$ at $95^{\circ} \mathrm{C}\left(10^{\prime}\right), 94^{\circ} \mathrm{C}\left(1^{\prime}\right), 56^{\circ} \mathrm{C}$ $\left(1^{\prime}\right)$ and $72^{\circ} \mathrm{C}\left(2^{\prime}\right)$. The analysis of RANKL and OPG mRNA levels were also determined in all the lesions (also by RealTimePCR using TaqMan chemistry), in order to categorize each sample in putative active and inactive lesions based on the RANKL/ OPG ratio as previously described ${ }^{29}$. The results are depicted as the relative level of gene expression; calculated in reference to internal controls GAPDH and $\beta$-actin expression in each sample using the $2^{-\mathrm{DD}}$ Ct method ${ }^{2,16}$.

\section{Data analysis}

Cytokine expression data is presented as a mean of mRNA expression, normalized by reference to the housekeeping genes from triplicate measurements in each sample. Comparisons among controls, inactive and active lesions were performed by ANOVA followed by Bonferroni post-test (performed in GraphPad Prism5.0 software, GraphPad Software Inc, San Diego, CA, USA); being $p<0.05$ was considered statistically significant. In order to examine possible clustering between the cytokines, a cluster analysis of 13 cytokine levels in 110 periapical granulomas was performed by using the Spearman rank correlation coefficient and the Kruskal-Wallis (KW) test ${ }^{44}$. After clusters determination, a hierarchical analysis was performed to access cytokine clusters association with activity/inactivity status, considering 1 ) the degree of lesion activity, ranked from inactivityto-activity based in the RANKL/OPG ratio, ranging from 0.43 to 4.46 , in accordance with the initially defined clusters; 2 ) the variance in individual cytokine expression levels within all 8 clusters were analyzed from the statistical viewpoint in order to generate a heat-like map representing the relative levels of expression (i.e. clusters with relative low levels are represented by the color white, clusters with relatively homogenous expression levels are represented by the color yellow, and clusters with increased gene expression are depicted in red; comprising a 6 grade scale representative of $16.66 \%$ within each sequential color/degree); 3 ) in the final ranking step the cytokines were ordered based in the cumulative frequency of high/intermediate/low levels along the inactive/active poles.

\section{RESULTS}

The pattern of cytokine expression in active and inactive granulomas

The mRNA levels expression of all targets investigated was found to be higher in total periapical granulomas when compared to controls (Figure 1). When lesions were compared based in the RANKL/OPG expression pattern ${ }^{29}, 40$ samples were found to be active (RANKL>OPG), while 70 presented an inactive lesion profile (RANKL $\leq O P G$ ) (Figure 1 ). When active and inactive lesions were compared, TNF- $\alpha$, IFN- $\gamma$, IL-17A and IL-21 mRNA levels were significantly higher in active granulomas (Figure 1), while in inactive lesions the expression levels of IL-4, IL-9, IL-10, IL-22 and FOXp3 were higher than in active granulomas (Figure 1 ). The levels of IL-23 and IL-33 in active and inactive lesions were similar from a statistical viewpoint (Figure 1).

Cluster and hierarchical analyzes of cytokine association with active and inactive lesions

In the view of the distinctly divergent cytokine profile between controls and lesions, cluster analysis was only performed with the lesion data in order to avoid biased associations. Initial cluster analysis performed with all (active + inactive) the lesions (Figure 2 ) resulted in the identification of 2 major clusters, comprised by 41 and 69 samples, presenting a $98 \%$ match with the clustering based in the RANKL/OPG patterns ${ }^{29}$. Considering such clear dichotomy, we performed additional cluster analysis within active and inactive lesions groups. In inactive lesions group (Table 1), 5 clusters were identified, being the variance in the expression levels of IL-17, IL-10, FOXp3, IFN- $\gamma$, IL-9, IL-33 and 


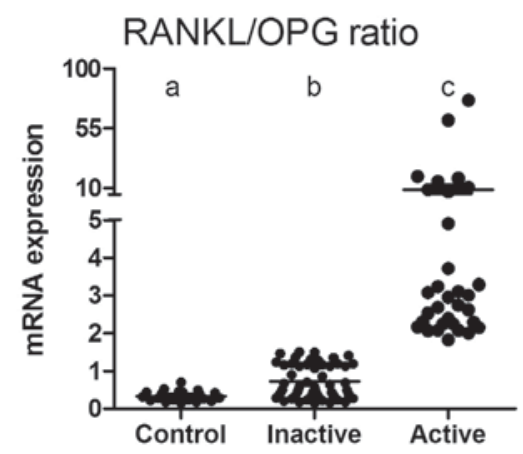

TNF-alpha (pro-inflammatory)

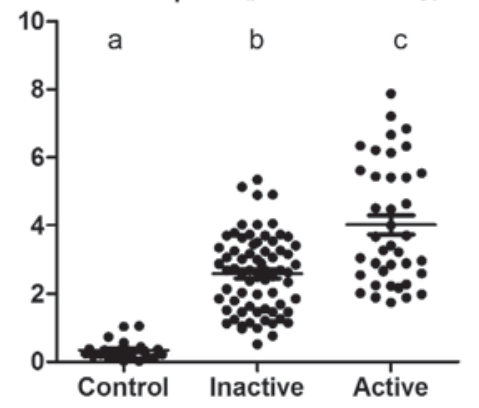

IL-23 (Th17 marker)

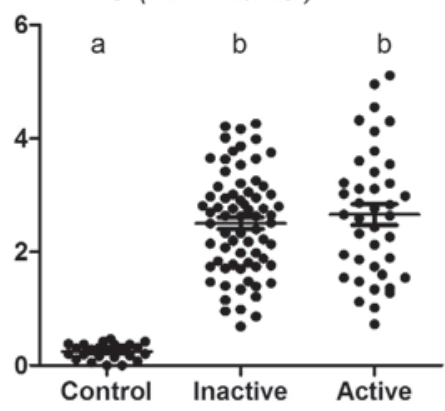

IL-33 (Th2 marker)

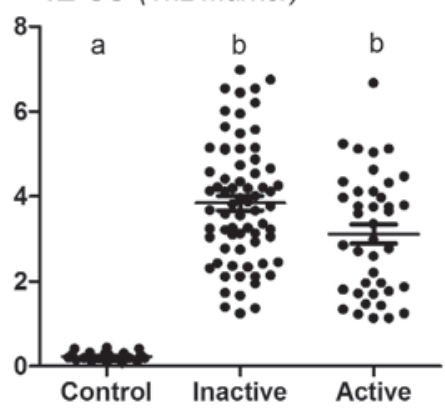

IL-10 (Tregs \& Tr1 marker)

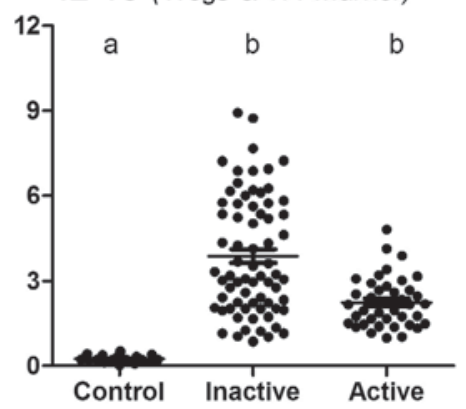

IFN-gamma (Th1 marker)

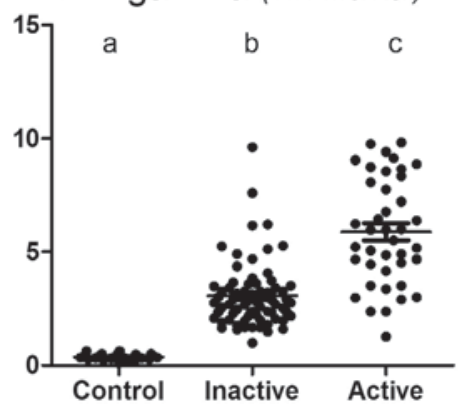

IL-21 (ThF marker)

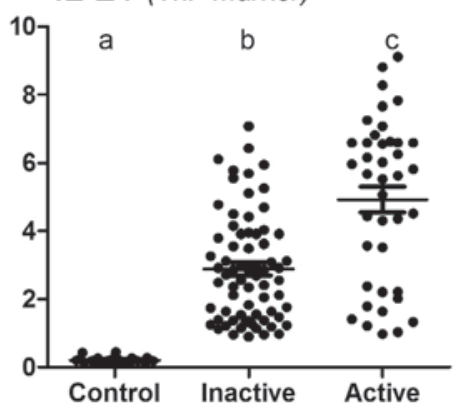

IL-9 (Th9 marker)

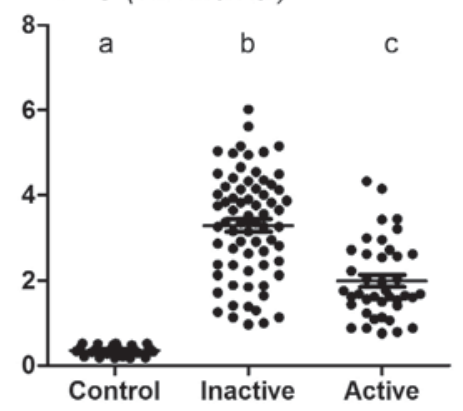

FOXp3 (Tregs marker)

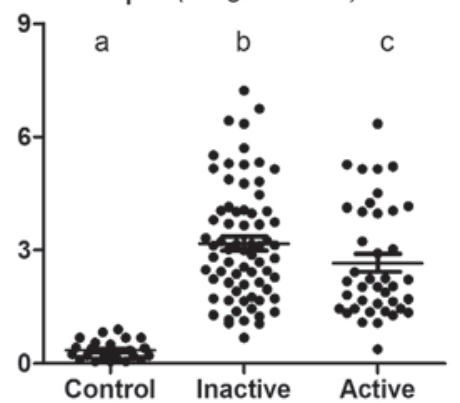

Figure 1 - Expression of individual mRNAs, with normalization to housekeeping genes, in periapical granulomas. Total RNA was extracted from periapical granulomas (experimental groups, $\mathrm{N}=110$ ) and periodontal ligament (control group, $\mathrm{N}=26$ ), and levels of RANKL and OPG mRNA were measured quantitatively by RealTimePCR using TaqMan chemistry. Based in profile of RANKL/OPG expression29 the lesions were then categorized into active (RANKL>OPG) or inactive (RANKL»OPG and RANKL<OPG). Different letters $(a, b, c)$ represent statistically significant differences among the respective groups $\left(\mathrm{P}<0.05\right.$; One-way ANOVA, Bonferroni post-test); ${ }^{*} \mathrm{P}<0.05$ (unpaired t-test) represent statistically significant differences between controls and periapical lesions (active + inactive) 
IL-4 statistically significant $(\mathrm{KW} \mathrm{p}<0.05)$ among the clusters of inactive lesions, while IL-22, OPG, IL-23, TNF- $\alpha$, RANKL and IL-21 levels presented $\mathrm{KW} \mathrm{p}$ values higher than 0.05 . When active lesions were analyzed (Table 2), 3 clusters were identified,

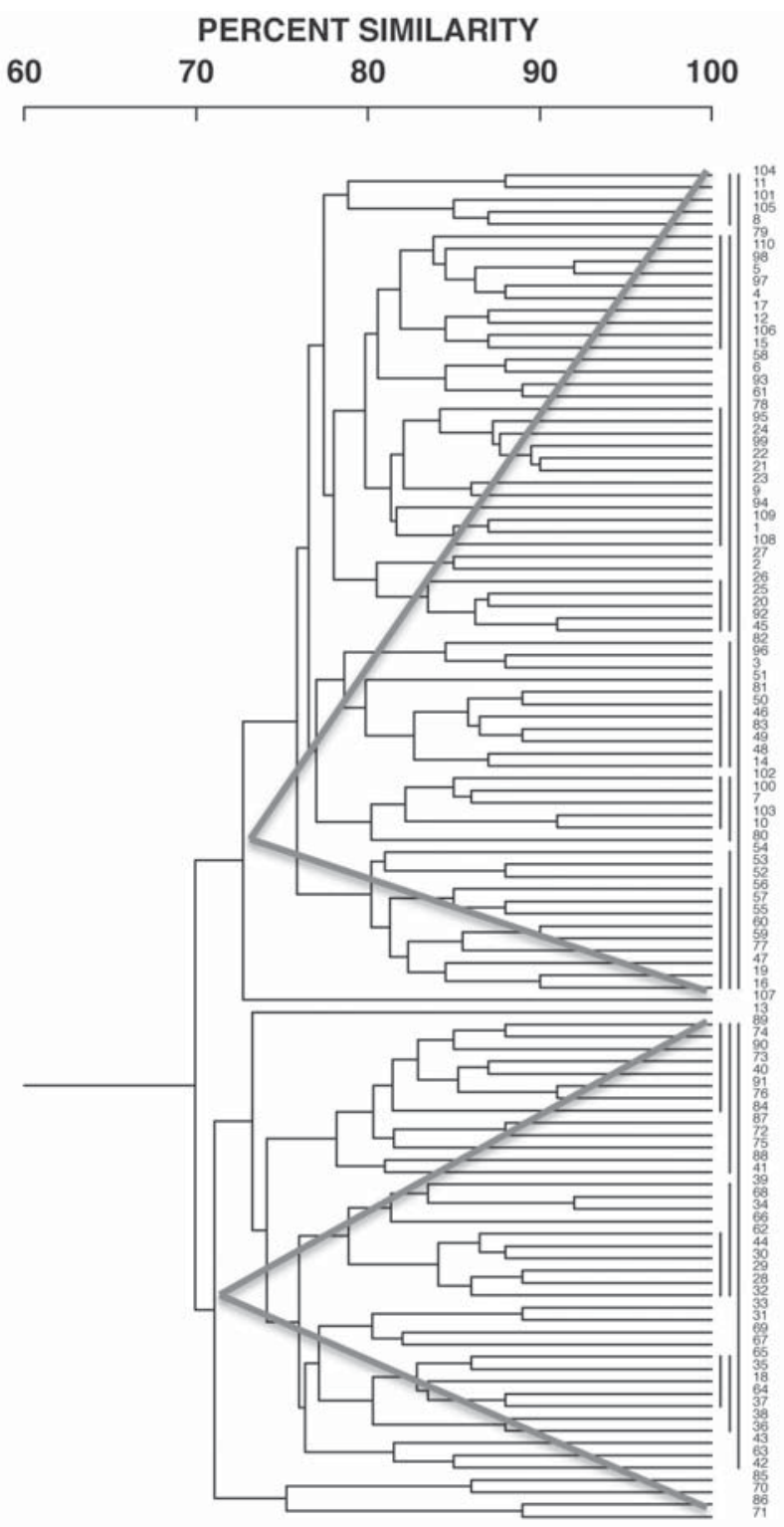

Figure 2- Patterns of cytokine expression in active and inactive periapical granulomas. Total RNA was extracted from periapical granulomas $(\mathrm{N}=110)$ and periodontal ligament control samples $(\mathrm{N}=26)$, and levels of TNF$\alpha$ (classic pro-inflammmatory cytokine), IL-10 (Treg and Tr1 marker), IFN- $\gamma$ (Th1 marker), IL-4 (Th2 marker), FOXp3 (Treg marker), CTLA4 (Treg marker), TGF- $\beta$ (Treg and Th3 marker), IL-9 (Th9 marker), IL-17A (Th17 marker), IL-17F (Th17 marker), IL-21 (Th17 or Tfh marker), IL-23 (Th17 marker) and IL-22 (Th22 marker) were measured quantitatively by RealTimePCR using TaqMan chemistry. Based on the profile of RANKL/OPG expression29, the lesions were then categorized into active (RANKL>OPG) or inactive (RANKL $\approx O P G$ and $R A N K L<O P G$ ) being the variance in the expression levels of IL22, IL-10, IFN- $\gamma$, IL-17, IL-33, FOXp3, IL-21 and RANKL statistically significant $(\mathrm{KW} p<0.05)$ among the active lesions clusters, while IL-9, IL-4, TNF- $\alpha$, OPG and IL-23 levels presented KW $p$ values higher than 0.05 .

The subsequent hierarchical analysis (Table 3 and Figure 3 ) ordered the samples regarding the activity level, from inactivity to activity ends, and demonstrated that inactivity pole was characterized by the highest OPG levels, sequentially followed in a downward way by FOXp3, IL-10, IL-9, IL-4 and IL-22. OPG and IL-22 expression profiles were relatively stable within inactive clusters; FOXP3 and IL-10 levels prevail in the clusters located in the inactivity pole edge, while a significant variation in the expression of IL-4 and IL-9 was verified in specific clusters (Table 3 and Figure 3 ). On the other hand, the lesion activity pole was characterized by the highest expression of TNF- $\alpha$, downward followed by RANKL, IL-21, IL-17 and IFN- $\gamma$ (Table 3 and Figure 3). High levels of TNF- $\alpha$ expression were a hallmark of all active clusters, RANKL expression prevails in the clusters located in the activity pole edge; also, a fairly specificity in the IFN- $\gamma$, IL-17 and IL-21 expression peaks was verified in definite clusters within active lesions (Table 3 and Figure 3). Interestingly, one cluster from inactive lesions subset presented a relatively high expression of IL-17. In an intermediate level within inactivity and activity poles, the cytokines IL-23 and IL-33 were not significantly associated with lesions' status (Table 3 and Figure 3).

\section{DISCUSSION}

Regulatory molecules such as cytokines play a key role in the pathogenesis periapical lesions ${ }^{12,19}$. Since the fragmented analysis of Th1, Th17, Th2, Th9, Th22 and Tregs related cytokines/markers expression suggests its involvement in periapical lesion development, but do not allow the analysis of the overall cytokine network operating in periapical environment; in this study we simultaneously investigated the patterns of such factors expression in active and inactive periapical lesions, as well the possible existence of cytokine clusters that could account for lesions outcome.

When the global cytokine expression profile in periapical lesions was compared with healthy control tissues, the expression of all cytokines/ markers investigated was found to be significantly augmented in the lesions, in accordance with previous studies ${ }^{1,6,9,10,19}$. In a general context, the widespread cytokine expression in the lesions is interpreted as a reflex of the chronic host response to the unremitting infection in the root canal and periapical area ${ }^{12,19}$. While the dichotomous 
comparison between health and disease conditions can be fairly revealing, it does not provide a disease severity and activity gradient, limiting the strength of such data to support more robust hypotheses. However, the comparison between active and inactive lesions ${ }^{29,48}$ provides a better picture of clinical variance, and therefore provide the support necessary to more robust analyses.

Initially, considering the active lesions scenario, the expression of TNF- $\alpha$, IFN- $\gamma$, IL-17 and IL-21 prevail in these lesions. TNF- $\alpha$ is classically described as a pro-inflammatory and osteoclastogenic cytokine ${ }^{13,18}$. Indeed, TNF- $\alpha$ was one of the first bone resorptive mediators identified in human periapical lesions, being its involvement in experimental periapical lesions progression clearly demonstrated in a cause-effect experiments ${ }^{18}$. Our data also demonstrated that the Th1-signature cytokine IFN-g was highly expressed in active lesions. While IFN-g is described to inhibit osteoclastogenesis in

Table 1- Five clusters (C1-C5) were identified in the inactive lesions group, being the variance in the expression levels of IL-17, IL-10, FOXp3, IFN- $\gamma$, IL-9, IL-33 and IL-4 statistically significant (KW p<0.05) among the clusters, while IL-22, OPG, IL-23, TNF- $\alpha$, RANKL and IL-21 levels presented KW p values higher than 0.05

\begin{tabular}{cccccccc}
\hline & inactive C1 & inactive C2 & inactive C3 & inactive C4 & inactive C5 & KW p & ANOVA p \\
\hline IL-17 & $4.10 \pm 1.34$ & $2.60 \pm 1.00$ & $4.98 \pm 0.91$ & $2.70 \pm 1.11$ & $1.75 \pm 0.79$ & 0.00000 & 0.00000 \\
IL-10 & $3.07 \pm 1.90$ & $5.79 \pm 1.60$ & $3.82 \pm 1.30$ & $2.12 \pm 0.74$ & $2.16 \pm 1.11$ & 0.00000 & 0.00000 \\
\hline FOXp3 & $3.46 \pm 2.47$ & $4.36 \pm 1.32$ & $2.42 \pm 0.66$ & $2.10 \pm 0.83$ & $2.53 \pm 1.07$ & 0.00000 & 0.00000 \\
IFN & $2.75 \pm 1.48$ & $2.76 \pm 0.95$ & $1.93 \pm 0.68$ & $3.03 \pm 0.52$ & $5.58 \pm 1.96$ & 0.00000 & 0.00000 \\
IL-9 & $3.03 \pm 1.88$ & $3.45 \pm 0.80$ & $2.69 \pm 1.15$ & $4.21 \pm 0.97$ & $1.84 \pm 0.86$ & 0.00011 & 0.00000 \\
IL-33 & $2.24 \pm 0.52$ & $3.70 \pm 1.32$ & $3.54 \pm 1.48$ & $4.84 \pm 1.26$ & $3.53 \pm 1.30$ & 0.00157 & 0.00146 \\
IL4 & $2.03 \pm 0.71$ & $3.13 \pm 1.31$ & $1.97 \pm 0.75$ & $2.76 \pm 1.12$ & $3.81 \pm 0.84$ & 0.00252 & 0.00244 \\
IL-22 & $4.18 \pm 1.11$ & $2.94 \pm 0.88$ & $3.07 \pm 0.60$ & $2.58 \pm 1.02$ & $2.78 \pm 0.92$ & 0.09754 & 0.01919 \\
OPG & $1.07 \pm 0.54$ & $0.78 \pm 0.41$ & $0.93 \pm 0.49$ & $0.80 \pm 0.41$ & $0.60 \pm 0.26$ & 0.40770 & 0.27883 \\
IL-23 & $2.15 \pm 0.97$ & $2.64 \pm 0.93$ & $2.12 \pm 0.74$ & $2.68 \pm 0.85$ & $2.48 \pm 1.01$ & 0.42409 & 0.40102 \\
\hline TNF $\alpha$ & $1.91 \pm 0.55$ & $2.59 \pm 0.98$ & $2.92 \pm 0.68$ & $2.56 \pm 1.49$ & $2.61 \pm 1.42$ & 0.49012 & 0.60985 \\
RANKL & $0.47 \pm 0.39$ & $0.38 \pm 0.18$ & $0.47 \pm 0.29$ & $0.43 \pm 0.18$ & $0.57 \pm 0.34$ & 0.76167 & 0.36040 \\
\hline IL21 & $2.31 \pm 0.96$ & $3.03 \pm 1.88$ & $2.90 \pm 1.35$ & $3.07 \pm 1.57$ & $2.39 \pm 1.10$ & 0.89569 & 0.73603 \\
N & 5 & 27 & 11 & 18 & 9 & nd & nd \\
\hline
\end{tabular}

Table 2- Three clusters (C1-C3) where identified in the active lesions group, being the variance in the expression levels of IL-22, IL-10, IFN- $\gamma$, IL-17, IL-33, FOXp3, IL-21 and RANKL statistically significant (KW p<0.05) among the clusters, while IL-9, IL-4, TNF- $\alpha$, OPG and IL-23 levels presented KW $p$ values higher than 0.05

\begin{tabular}{cccccc}
\hline & active C1 & active C2 & active C3 & KW p & ANOVA p \\
\hline IL-22 & $1.13 \pm 0.30$ & $3.30 \pm 0.79$ & $1.45 \pm 0.77$ & 0.00007 & 0.00000 \\
\hline IL-10 & $1.71 \pm 0.44$ & $2.36 \pm 0.61$ & $3.10 \pm 0.98$ & 0.00022 & 0.00001 \\
\hline IFN & $5.72 \pm 1.43$ & $3.62 \pm 2.41$ & $8.02 \pm 1.84$ & 0.00029 & 0.00002 \\
IL-17 & $4.74 \pm 1.74$ & $5.54 \pm 0.75$ & $3.14 \pm 0.92$ & 0.00073 & 0.00111 \\
\hline IL-33 & $3.85 \pm 1.18$ & $1.88 \pm 1.25$ & $2.80 \pm 1.19$ & 0.00135 & 0.00069 \\
\hline FOXp3 & $1.98 \pm 1.04$ & $3.08 \pm 1.51$ & $3.55 \pm 1.68$ & 0.01007 & 0.00902 \\
\hline IL21 & $3.88 \pm 2.48$ & $6.07 \pm 2.49$ & $5.91 \pm 1.09$ & 0.01426 & 0.01684 \\
RANKL & $1.43 \pm 0.65$ & $1.16 \pm 0.44$ & $0.86 \pm 0.35$ & 0.03475 & 0.02563 \\
\hline IL-9 & $1.73 \pm 0.77$ & $1.80 \pm 0.58$ & $2.60 \pm 1.12$ & 0.05429 & 0.02585 \\
IL4 & $2.31 \pm 1.14$ & $1.48 \pm 0.47$ & $2.36 \pm 1.16$ & 0.15877 & 0.10883 \\
\hline TNF $\alpha$ & $4.34 \pm 1.80$ & $3.76 \pm 1.97$ & $3.63 \pm 1.56$ & 0.40797 & 0.50789 \\
OPG & $0.32 \pm 0.23$ & $0.36 \pm 0.16$ & $0.30 \pm 0.19$ & 0.55426 & 0.77663 \\
\hline IL-23 & $2.74 \pm 0.88$ & $2.57 \pm 1.52$ & $2.60 \pm 1.27$ & 0.68514 & 0.91416 \\
N & 20 & 9 & 11 & nd & nd \\
\hline
\end{tabular}


vitro, a clear association with increased bone loss is described in vivo, where the upregulation of TNF- $\alpha$, IL-1 $\beta$ and RANKL overcome the direct antiosteoclastogenic effect described in vitro $11,14,22,34,35$. Regarding periapical lesions, IFN- $\gamma$ positive cells are found in both periapical granulomas and cysts, where they are supposed to be involved in periapical lesion development ${ }^{6}$. Additionally, the Th17-prototytical cytokine IL-17A, described as a potent inflammatory and osteoclastogenic factor, was also found in higher levels in active periapical lesions where it is supposed to exacerbate the inflammatory osteolytic process $7,28,33,41$. The last cytokine found to be overexpressed in active lesions is IL-21, a cytokine produced by Th17 and Tfh cells previously implicated in osteoclastogenesis and bone destruction ${ }^{8,24}$. Tfh, a CD4+ Tcell subset found in the B-cell follicles of secondary (and feasibly tertiary) lymphoid organs ${ }^{27}$, is described as a major contributor to $\mathrm{B}$ cell-mediated antibody responses and an important source of IL- $21^{43}$. Considering the chronic nature of periapical lesions and the abundant presence of B cells in such environment ${ }^{26}$, it would be possible to suggest that IL-21 in periapical area contributes to a Thf-B cell response axis, similarly to what was described in tertiary lymphoid tissues associated with chronic infection sites ${ }^{20,47}$. Since $B$ cells are described as a potential RANKL source ${ }^{12,19}$, Thf-B cell axis can directly drive lesions activity via RANKL production.

Taken together, the discussed evidences suggest a role of TNF- $\alpha$, IFN- $\gamma$, IL-17A and IL-21 in the periapical lesions progression. In order to clarify the possible interplays between such cytokines, cluster hierarchical analyses were performed and 3 distinct cytokine clusters were identified in active lesions subset. The maximum activity (i.e. higher RANKL/OPG ratio) cluster (active cluster 1 ) was characterized by the highest TNF- $\alpha$ and RANKL levels among active lesions, and relatively high IL-21, IL-17 and IFN- $\gamma$ expression. In this high activity scenario, it is possible to suggest that local TNF- $\alpha$ and IL-21 activity favors the infiltration and activity of both Th1 and Th17 cells, as previously described in experimental arthritis ${ }^{32}$. Accordingly, when mice strains with opposing bone resorption susceptibility phenotypes were compared, parallel high levels of TNF- $\alpha$ and IFN- $\gamma$ were associated with increased bone resorption activity ${ }^{45,46}$. However, a negative correlation between the relatively high levels of both IFN- $\gamma$ and IL-17 was observed in all the active lesion clusters. Indeed, active cluster 2 was characterized by the highest levels of IL-21 and IL-17, while the active cluster 3 presented the highest IFN-g levels and a high IL-21 expression. Therefore, Th1- (active cluster 3) and Th17- (active cluster 2) biased clusters were evidenced within active clusters. Accordingly, previous studies demonstrate a reciprocal Th1/Th17 inhibition, suggesting that Th1 and Th17 mediators may be independently associated to the progression of inflammatory osteolytic lesions ${ }^{4}$. It is essential to consider that the hierarchical analysis points to Th1-biased cluster (active cluster 3) as lower activity cluster than the clusters with high Th17 activity (active clusters 1 and 2). Accordingly, while literature seems to present a relative consensus regarding the osteoclastogenic role of IL-17/Th17, the association of IFN- $\gamma /$ Th1 with bone resorption process remains quite controversial ${ }^{12,19}$. Regarding IL-21, the levels of such cytokine were found to be elevated in both Th1- and Th17-biased clusters, but no direct positive/negative correlations were observed, suggesting that Tfh cells may operate in parallel (or even cooperatively) with both Th1 and Th17 mediators.

Table 3- Hierarchical analysis ordering the samples regarding the activity level, from inactivity to activity ends based on the RANKL/OPG profile

\begin{tabular}{|c|c|c|c|c|c|c|c|c|}
\hline lesion status & inactivity & & & & & & & activity \\
\hline RANKL/OPG & 0.43 & 0.48 & 0.50 & 0.53 & 0.95 & 2.8 & 3.22 & 4.46 \\
\hline TNF $\alpha$ & $1.91 \pm 0.55$ & $2.59 \pm 0.98$ & $2.92 \pm 0.68$ & $2.56 \pm 1.49$ & $2.61 \pm 1.42$ & $3.63 \pm 1.56$ & $3.76 \pm 1.97$ & $4.34 \pm 1.80$ \\
\hline RANKL & $0.47 \pm 0.39$ & $0.38 \pm 0.18$ & $0.47 \pm 0.29$ & $0.43 \pm 0.18$ & $0.57 \pm 0.34$ & $0.86 \pm 0.35$ & $1.16 \pm 0.44$ & $1.43 \pm 0.65$ \\
\hline IL21 & $2.31 \pm 0.96$ & $3.03 \pm 1.88$ & $2.90 \pm 1.35$ & $3.07 \pm 1.57$ & $2.39 \pm 1.10$ & $5.91 \pm 1.09$ & $6.07 \pm 2.49$ & $3.88 \pm 2.48$ \\
\hline IL-17 & $4.10 \pm 1.34$ & $2.60 \pm 1.00$ & $4.98 \pm 0.91$ & $2.70 \pm 1.11$ & $1.75 \pm 0.79$ & $3.14 \pm 0.92$ & $5.54 \pm 0.75$ & $4.74 \pm 1.74$ \\
\hline IFN & $2.75 \pm 1.48$ & $2.76 \pm 0.95$ & $1.93 \pm 0.68$ & $3.03 \pm 0.52$ & $5.58 \pm 1.96$ & $8.02 \pm 1.84$ & $3.62 \pm 2.41$ & $5.72 \pm 1.43$ \\
\hline IL-33 & $2.24 \pm 0.52$ & $3.70 \pm 1.32$ & $3.54 \pm 1.48$ & $4.84 \pm 1.26$ & $3.53 \pm 1.30$ & $2.80 \pm 1.19$ & $1.88 \pm 1.25$ & $3.85 \pm 1.18$ \\
\hline IL-23 & $2.15 \pm 0.97$ & $2.64 \pm 0.93$ & $2.12 \pm 0.74$ & $2.68 \pm 0.85$ & $2.48 \pm 1.01$ & $2.60 \pm 1.27$ & $2.57 \pm 1.52$ & $2.74 \pm 0.88$ \\
\hline IL-22 & $4.18 \pm 1.11$ & $2.94 \pm 0.88$ & $3.07 \pm 0.60$ & $2.58 \pm 1.02$ & $2.78 \pm 0.92$ & $1.45 \pm 0.77$ & $3.30 \pm 0.79$ & $1.13 \pm 0.30$ \\
\hline IL4 & $2.03 \pm 0.71$ & $3.13 \pm 1.31$ & $1.97 \pm 0.75$ & $2.76 \pm 1.12$ & $3.81 \pm 0.84$ & $2.36 \pm 1.16$ & $1.48 \pm 0.47$ & $2.31 \pm 1.14$ \\
\hline IL-9 & $3.03 \pm 1.88$ & $3.45 \pm 0.80$ & $2.69 \pm 1.15$ & $4.21 \pm 0.97$ & $1.84 \pm 0.86$ & $2.60 \pm 1.12$ & $1.80 \pm 0.58$ & $1.73 \pm 0.77$ \\
\hline IL-10 & $3.07 \pm 1.90$ & $5.79 \pm 1.60$ & $3.82 \pm 1.30$ & $2.12 \pm 0.74$ & $2.16 \pm 1.11$ & $3.10 \pm 0.98$ & $2.36 \pm 0.61$ & $1.71 \pm 0.44$ \\
\hline FOXp3 & $3.46 \pm 2.47$ & $4.36 \pm 1.32$ & $2.42 \pm 0.66$ & $2.10 \pm 0.83$ & $2.53 \pm 1.07$ & $3.55 \pm 1.68$ & $3.08 \pm 1.51$ & $1.98 \pm 1.04$ \\
\hline OPG & $1.07 \pm 0.54$ & $0.78 \pm 0.41$ & $0.93 \pm 0.49$ & $0.80 \pm 0.41$ & $0.60 \pm 0.26$ & $0.30 \pm 0.19$ & $0.36 \pm 0.16$ & $0.32 \pm 0.23$ \\
\hline
\end{tabular}




\section{ACTIVE LESIONS RELATED MARKERS}
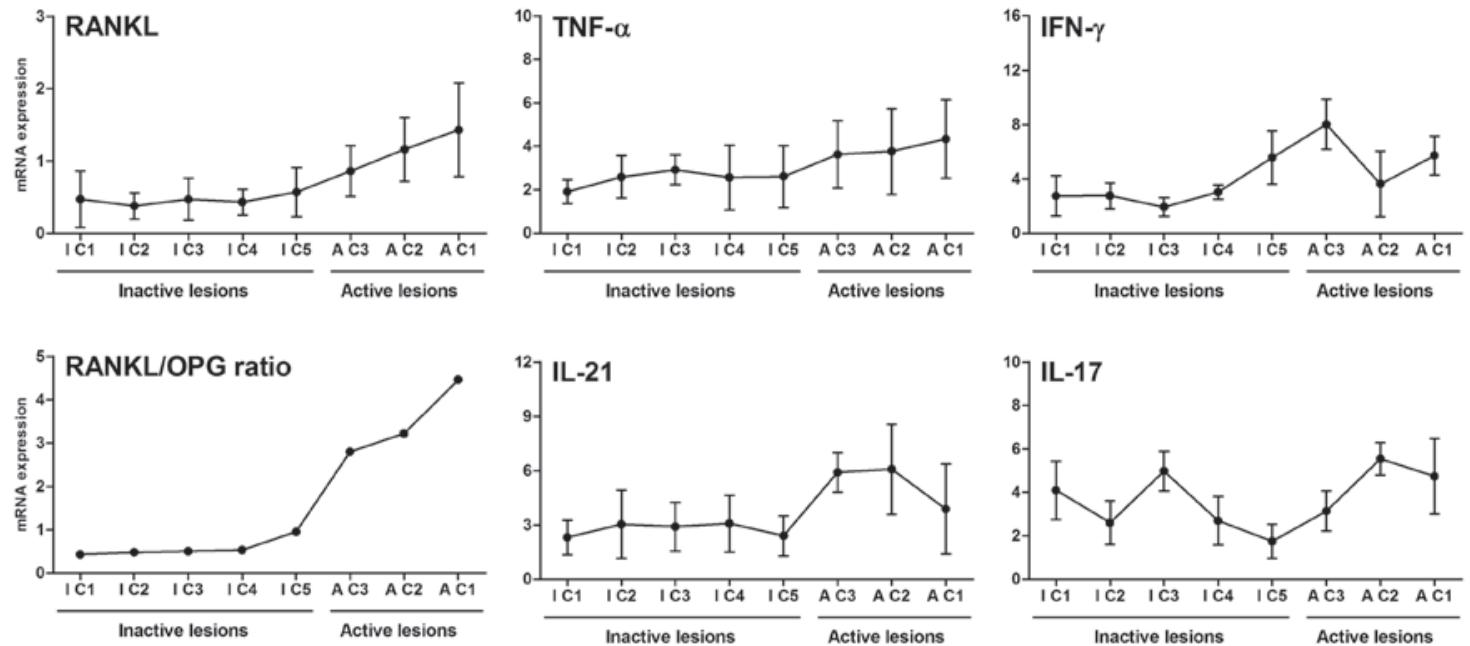

\section{INACTIVE LESIONS RELATED MARKERS}
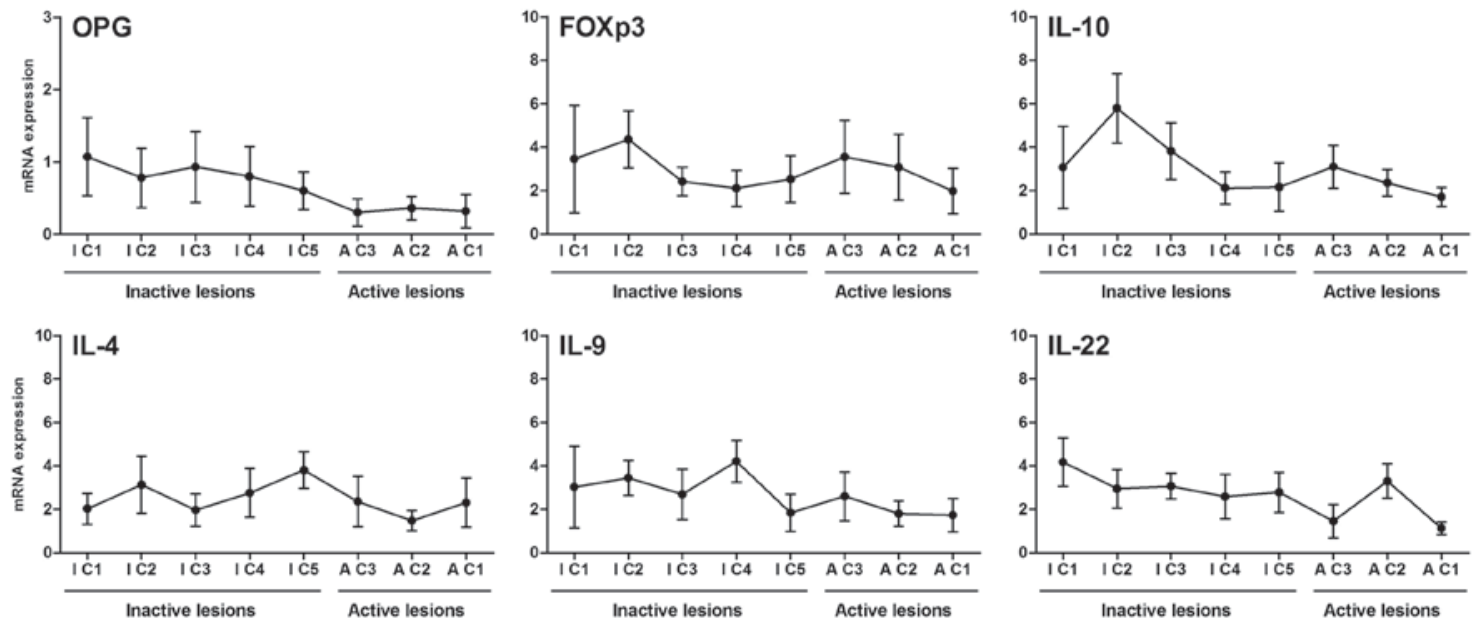

\section{UNRELATED MARKERS}
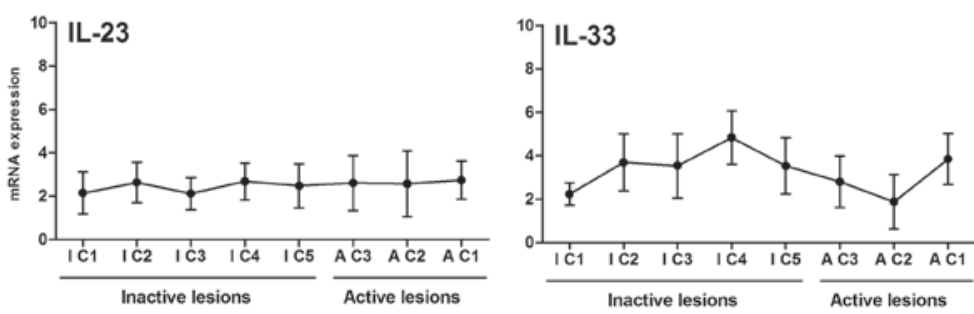

Figure 3- Patterns of cytokine expression in the clusters associated with active and inactive periapical granulomas nature. Hierarchical analysis demonstrated that the inactivity pole was characterized by the highest OPG levels, sequentially followed in a downward way by FOXp3, IL-10, IL-9, IL-4 and IL-22. OPG and IL-22 expression profiles were relatively stable within inactive clusters; FOXP3 and IL-10 levels prevail in the clusters located in the inactivity pole edge, while a significant variation in expression of IL-4 and IL-9 was verified in specific clusters. On the other hand, the lesions activity pole was characterized by the highest expression of TNF- $\alpha$, downward followed by RANKL, IL-21, IL-17 and IFN- $\gamma$. High levels of TNF- $\alpha$ expression were a hallmark of all active clusters, and RANKL expression prevail in the clusters located in the activity pole edge; also, a fairly specificity in the IFN- $\gamma$, IL-17 and IL-21 expression peaks was verified in definite clusters within active lesions. Interestingly, one cluster from inactive lesions subset, presented a relatively high expression of IL-17. The cytokines IL-23 and IL-33 were not significantly associated with lesions' status 
Moving the focus towards the putative determinants of lesions inactivity, IL-4, FOXp3, IL10, IL-9 and IL-22 levels in inactive lesions overcome the levels observed in active sites. IL-4 represents the prototypic Th2 cytokine, being a potential protective mediator due its ability to upregulate OPG levels and suppress pro-inflammatory responses ${ }^{40}$. Besides IL-4, IL-33 also has been associated with Th2 responses and presents similar properties towards bone protective action, such as the inhibition of osteoclast differentiation ${ }^{39}$. Along Th2 responses, Tregs (characterized by the expression of FOXp3) and $\operatorname{Tr} 1$ cells are supposed to attenuate periapical lesions development ${ }^{6,9}$. Indeed, IL-10 (a characteristic product of both Tregs and Tr1 subsets) was previously detected in periapical lesions, where it inhibits inflammatory cells influx and bone resorption ${ }^{30,38}$. While the positive correlations observed between IL-10 and FOXp3 levels in inactive lesions reinforce that Tregs can be a significant source of IL-10 in periapical area, the lack of definitive $\operatorname{Tr} 1$ markers does not allow stronger assumptions regarding its possible involvement in this system. Besides IL-4 and IL-10, IL-9 and IL-22 were found to be overexpressed in inactive lesions, in accordance with previous data ${ }^{1}$. Th9 cells have been described to present an interesting plasticity, acting together with Th2 in some inflammatory processes or exerting immunosuppressive actions via IL-10 production ${ }^{42}$. IL-22 is also highly pleiotropic, since it can cooperate with IL-10 in a regulatory network that reduces the severity of experimental arthritis ${ }^{37}$ or exerts pro-inflammatory effects by a synergistic action with classic pro-inflammatory mediators such as TNF- $\alpha$ of $\mathrm{IL}-17^{49}$.

When potentially protective mediators associated with periapical lesions inactivity are scrutinized by the cluster hierarchical analysis, 5 distinct clusters were observed. The lowest activity pole is comprised by a cluster (inactive cluster 1 ) presenting the highest levels of OPG and IL-22, in parallel with relatively high levels of IL-10 and FOXP3 (the Tregs hallmark), comprising therefore a Th22/Tregs-biased cluster. While IL-22 can be a product of Th17 cells and operates in concert with IL-17 in inflammatory and autoimmune diseases ${ }^{51}$, recent evidences demonstrate that when produced by Th2 2 cells in an milieu with low IL-17 levels (such as this specific cluster), IL-22 induce IL-10mediated immunosuppressive effects ${ }^{31}$. Also, since Tregs are able to suppress Th17/IL-17-mediated responses ${ }^{50}$, it is possible to suggest that Tregs favor IL-22/IL-10 axis via Th17 suppression. The subsequent cluster in a presumed ascending activity level (inactive cluster 2) is characterized by the highest levels of FOXp3 and IL-10, along relatively high levels of both IL-4 and IL-9; typifying a Treg/
Tr1-biased cluster. The classic description of Tregs and $\operatorname{Tr} 1$ cells as significant sources of IL-10 was previously discussed, and supports the dominance of Tregs and $\operatorname{Tr} 1$ in determining such cluster inactivity via IL-10 production. Interestingly, it was recently demonstrated that the presence of FOXP3 is sufficient to suppress the expression of IL-22 21 , which could account for the relatively low levels of IL-22 observed in this cluster. While such association contradicts the hypothesis of the Th22/ Tregs- cluster previously discussed, we can consider that the relatively high IL-4 and IL- 9 levels may be a reflex of a distinct $T$ cell polarization in this cluster. Indeed, both Th2 responses (via Th2-chemokine CCL22 mediated Tregs chemoattraction) and IL-10 can contribute to the immunosuppressive response via IL-10 17,42 .

The next inactive lesions cluster (inactive cluster 3 ) is characterized by a high IL-10 expression, relatively low FOXp3, IL-4 and IL-9 levels, and a singular high IL-17 expression. Initially considering the lack of a direct correlation/association between FOXp3 levels and IL-10, it is possible to hypothesize a dominant role for Tr1 instead of classic FOXp3+ Tregs in this cluster. Indeed, the high levels of IL17 in parallel with the low levels of FOXp3 may be representative of a plastic behavior of Th17/ Tregs cells, where environmental signals can limit Tregs suppressive activity ${ }^{52}$. Following the clusters ascending activity level, the subsequent cluster (inactive cluster 4 ) is characterized by the highest IL-9 and IL-33 levels. Since no evidences of collaborative actions between IL-33 and IL- 9 are reported, it is reasonable to consider that these $T$ cell subsets may exert independent roles in the determination of lesions inactivity. While the data regarding IL-9 and bone lytic process is scarce as previously discussed, its possible association with lesions inactivity relies on the possible association with IL-10 production ${ }^{1,42}$. Considering the potential protective role of IL-33, while its antiosteoclastogenic action was recently described ${ }^{23}$, IL-33 levels are similar in overall inactive/active lesions, weakening the hypothesis that IL-33 plays a major role as a determinant of periapical lesions inactivity.

Finally, in the edge between inactive and active lesion clusters, a Th2-biased cluster (inactive cluster 5 ) is characterized by the highest IL-4 levels within inactive lesions. IL-4 is usually described to limit or attenuate the tissue damage due its antiinflammatory properties, which include the inhibition of RANKL, concomitantly with OPG upregulation ${ }^{36}$, and the suppression of pro-inflammatory and Th1type responses ${ }^{6,19,35}$. Recently, it was described that the Th2-type chemokine CCL22 (which can be induced by $\mathrm{IL}-4$ ) attenuates periodontal lesions severity though Tregs chemoattraction ${ }^{17}$. However, 
the relatively low levels of FOXp3 and IL-10 in this Th2 biased cluster, as well as the lack of negative correlations between IL-4 and IFN-g does not support such hypotheses. These results suggest that a dominant IL-4 response, in parallel to low levels of other potentially protective cytokines, may not be highly effective in determining lesions inactivity. Indeed, this IL-4-biased cluster is located in the frontier between inactive and active lesions, being its RANKL/OPG ratio roughly 2 times higher than the other 4 inactive clusters.

Taken together, our results demonstrate distinct patterns of cytokine expression in active and inactive periapical granulomas. In active lesions, pro-inflammatory Th1 and Th17 skewed clusters in concert with IL-21 are supposed to independently drive lesions progression. Conversely, inactive lesions present a more complex scenario, were Th22/Tregs, Tregs/Tr1, Tr1, Th9 and Th2 biased clusters can account for lesion inactivity status. However, further cause-and-effect studies are required to fully dissect the cytokine network involved in the pathogenesis of periapical lesions, aiming to unravel the protective and destructive pathways and therefore contribute to improve the diagnosis and treatment of these pathologies. However, further studies are required to support our hypothesis.

\section{CONCLUSION}

A clear dichotomy exists in the profile of cytokine expression in inactive and active periapical lesions. While the widespread cytokine expression seems to be a feature of such chronic lesions, hierarchical cluster analysis demonstrates the association of TNF- $\alpha$, IL-21, IL-17 and IFN- $\gamma$ (ordered by their supposed destructive potential) with lesions' activity, and the association of FOXp3, IL-10, IL-9, IL-4 and IL-22 (ordered in its supposed protective potential) with lesions' inactivity.

\section{ACKNOWLEDGMENTS}

The authors would like to thank Daniele Ceolin, Patricia Germino and Tania Cestari for their excellent technical assistance. This study was supported by grants and scholarships from FAPESP (2012/151333, 2013/05994-4) and CNPq (302712/2011-9). The authors deny any conflicts of interest related to this study.

\section{REFERENCES}

1- Aranha AM, Repeke CE, Garlet TP, Vieira AE, Campanelli AP, Trombone AP, et al. Evidence supporting a protective role for th9 and th22 cytokines in human and experimental periapical lesions. J Endod. 2013;39(1):83-7.

2- Araujo-Pires AC, Biguetti CC, Rodini CO, Campanelli AP, Trombone AP, Letra $A$, et al. Mesenchymal stem cells as active prohealing and immunosuppressive agents in periapical environment: evidences from human and experimental periapical lesions. J Endod. 2014. In Press.

3- Belkaid Y, Tarbell K. Regulatory T cells in the control of hostmicroorganism interactions. Ann Rev Immunol. 2009;27:551-89. 4- Brand S. Crohn's disease: Th1, Th17 or both? The change of a paradigm: new immunological and genetic insights implicate Th17 cells in the pathogenesis of Crohn's disease. Gut. 2009;58(8):1152-67.

5- Cavalla F, Araujo-Pires AC, Biguetti CC, Garlet GP. Cytokine networks regulating inflammation and immune defense in the oral cavity. Curr Oral Health Rep. 2014. In press.

6- Colić M, Gazivoda D, Vucević D, Vasilijić S, Rudolf R, Lukić A. Proinflammatory and immunoregulatory mechanisms in periapical lesions. Mol Immunol. 2009;47(1):101-13.

7- Colić M, Vasilijić S, Gazivoda D, Vucević D, Marjanović M, Lukić A. Interleukin-17 plays a role in exacerbation of inflammation within chronic periapical lesions. Eur J Oral Sci. 2007;115(4):31520.

8- Dutzan N, Vernal R, Vaque JP, García-Sesnich J, Hernandez M, Abusleme $L$, et al. Interleukin-21 expression and its association with proinflammatory cytokines in untreated chronic periodontitis patients. J Periodontol. 2012;83(7):948-54.

9- Fukada SY, Silva TA, Garlet GP, Rosa AL, Silva JS, Cunha FQ. Factors involved in the T helper type 1 and type 2 cell commitment and osteoclast regulation in inflammatory apical diseases. Oral Microbiol Immunol. 2009;24(1):25-31.

10- Fukada SY, Silva TA, Saconato IF, Garlet GP, Avila-Campos MJ, Silva JS, et al. iNOS-derived nitric oxide modulates infectionstimulated bone loss. J Dent Res. 2008;87(12):1155-9.

11- Gao Y, Grassi F, Ryan MR, Terauchi M, Page K, Yang X, et al. IFN-gamma stimulates osteoclast formation and bone loss in vivo via antigen-driven $\mathrm{T}$ cell activation. J Clin Invest. 2007;117(1):122-32.

12- Garlet GP. Destructive and protective roles of cytokines in periodontitis: a re-appraisal from host defense and tissue destruction viewpoints. J Dent Res. 2010;89(12):1349-63.

13- Garlet GP, Cardoso CR, Campanelli AP, Ferreira BR, AvilaCampos MJ, Cunha FQ, et al. The dual role of p55 tumour necrosis factor-alpha receptor in Actinobacillus actinomycetemcomitansinduced experimental periodontitis: host protection and tissue destruction. Clin Exp Immunol. 2007;147(1):128-38.

14- Garlet GP, Cardoso CR, Campanelli AP, Garlet TP, Avila-Campos MJ, Cunha FQ, et al. The essential role of IFN-gamma in the control of lethal Aggregatibacter actinomycetemcomitans infection in mice. Microbes Infect. 2008;10(5):489-96.

15- Garlet GP, Cardoso CR, Mariano FS, Claudino M, Assis GF, Campanelli AP, et al. Regulatory $T$ cells attenuate experimental periodontitis progression in mice. J Clin Periodontol. 2010;37(7):591-600.

16- Garlet GP, Horwat R, Ray HL Jr, Garlet TP, Silveira EM, Campanelli AP, et al. Expression analysis of wound healing genes in human periapical granulomas of progressive and stable nature. J Endod. 2012;38(2):185-90.

17- Glowacki AJ, Yoshizawa S, Jhunjhunwala S, Vieira AE, Garlet GP, Sfeir C, et al. Prevention of inflammation-mediated bone loss in murine and canine periodontal disease via recruitment of regulatory lymphocytes. Proc Natl Acad Sci USA. 2013;110(46):18525-30. 18- Graves D. Cytokines that promote periodontal tissue destruction. J Periodontol. 2008;79(8 Suppl):1585-91. 
19- Graves DT, Oates T, Garlet GP. Review of osteoimmunology and the host response in endodontic and periodontal lesions. J Oral Microbiol. 2011;3.

20- Huang HY, Luther SA. Expression and function of interleukin-7 in secondary and tertiary lymphoid organs. Semin Immunol. 2012;24(3):175-89.

21- Jeron A, Hansen W, Ewert F, Buer J, Geffers R, Bruder D. ChIP-on-chip analysis identifies IL-22 as direct target gene of ectopically expressed FOXP3 transcription factor in human T cells. BMC Genomics. 2012;13:705.

22- Ji JD, Park-Min KH, Shen Z, Fajardo RJ, Goldring SR, McHugh $\mathrm{KP}$, et al. Inhibition of RANK expression and osteoclastogenesis by TLRs and IFN-gamma in human osteoclast precursors. J Immunol. 2009;183(11):7223-33.

23- Keller J, Catala-Lehnen P, Wintges K, Schulze J, Bickert T, Ito W, et al. Transgenic over-expression of interleukin-33 in osteoblasts results in decreased osteoclastogenesis. Biochem Biophys Res Commun. 2012;417(1):217-22.

24- Kwok SK, Cho ML, Park MK, Oh HJ, Park JS, Her YM, et al. Interleukin-21 promotes osteoclastogenesis in humans with rheumatoid arthritis and in mice with collagen-induced arthritis. Arthritis Rheum. 2012;64(3):740-51.

25- Liu SM, King C. IL-21-producing Th cells in immunity and autoimmunity. J Immunol. 2013;191(7):3501-6.

26- Lukić A, Vasilijić S, Majstorović I, Vucević D, Mojsilović S, Gazivoda D, et al. Characterization of antigen-presenting cells in human apical periodontitis lesions by flow cytometry and immunocytochemistry. Int Endod J. 2006;39(8):626-36.

27- MacLennan IC. Germinal centers. Annu Rev Immunol. 1994;12:117-39.

28- Marçal JR, Samuel RO, Fernandes D, Araujo MS, Napimoga $\mathrm{MH}$, Pereira SA, et al. T-helper cell type 17/regulatory T-cell immunoregulatory balance in human radicular cysts and periapical granulomas. J Endod. 2010;36(6):995-9.

29- Menezes R, Garlet TP, Letra A, Bramante CM, Campanelli AP, Figueira RC, et al. Differential patterns of receptor activator of nuclear factor kappa B ligand/osteoprotegerin expression in human periapical granulomas: possible association with progressive or stable nature of the lesions. J Endod. 2008;34(8):932-8.

30- Menezes R, Garlet TP, Trombone AP, Repeke CE, Letra A, Granjeiro JM, et al. The potential role of suppressors of cytokine signaling in the attenuation of inflammatory reaction and alveolar bone loss associated with apical periodontitis. J Endod. 2008;34(12):1480-4.

31- Nakagome K, Imamura M, Kawahata K, Harada H, Okunishi $\mathrm{K}$, Matsumoto T, et al. High expression of IL-22 suppresses antigen-induced immune responses and eosinophilic airway inflammation via an IL-10-associated mechanism. J Immunol. 2011;187(10):5077-89.

32- Notley CA, Inglis JJ, Alzabin S, McCann FE, McNamee KE, Williams RO. Blockade of tumor necrosis factor in collagen-induced arthritis reveals a novel immunoregulatory pathway for Th1 and Th17 cells. J Exp Med. 2008;205(11):2491-7.

33- Oseko F, Yamamoto T, Akamatsu Y, Kanamura N, Iwakura Y, Imanishi J, et al. IL-17 is involved in bone resorption in mouse periapical lesions. Microbiol Immunol. 2009;53(5):287-94.

34- Repeke CE, Ferreira SB Jr, Claudino M, Silveira EM, Assis GF, Avila-Campos MJ, et al. Evidences of the cooperative role of the chemokines CCL3, CCL4 and CCL5 and its receptors CCR1+ and CCR5+ in RANKL+ cell migration throughout experimental periodontitis in mice. Bone. 2010;46(4):1122-30.

35- Repeke CE, Ferreira SB Jr, Vieira AE, Silveira EM, AvilaCampos MJ, Silva JS, et al. Dose-response met-RANTES treatment of experimental periodontitis: a narrow edge between the disease severity attenuation and infection control. PloS One. $2011 ; 6(7): e 22526$.
36- Saidenberg-Kermanac'h N, Bessis N, Lemeiter D, de Vernejoul MC, Boissier MC, Cohen-Solal M. Interleukin-4 cellular gene therapy and osteoprotegerin decrease inflammation-associated bone resorption in collagen-induced arthritis. J Clin Immunol. 2004;24(4):370-8.

37- Sarkar S, Zhou X, Justa S, Bommireddy SR. Interleukin-22 reduces the severity of collagen-induced arthritis in association with increased levels of interleukin-10. Arthritis Rheum. 2013;65(4):960-71.

38- Sasaki H, Hou L, Belani A, Wang CY, Uchiyama T, Müller R, et al. IL-10, but not IL-4, suppresses infection-stimulated bone resorption in vivo. J Immunol. 2000;165(7):3626-30.

39- Schett G. Effects of inflammatory and anti-inflammatory cytokines on the bone. Eur J Clin Invest. 2011;41(12):1361-6.

40- Stein NC, Kreutzmann C, Zimmermann SP, Niebergall U, Hellmeyer L, Goettsch C, et al. Interleukin-4 and interleukin-13 stimulate the osteoclast inhibitor osteoprotegerin by human endothelial cells through the STAT6 pathway. J Bone Miner Res. $2008 ; 23(5): 750-8$.

41- Takayanagi H. New developments in osteoimmunology. Nat Rev Rheumatol. 2012;8(11):684-9.

42- Tan C, Aziz MK, Lovaas JD, Vistica BP, Shi G, Wawrousek EF, et al. Antigen-specific Th9 cells exhibit uniqueness in their kinetics of cytokine production and short retention at the inflammatory site. J Immunol. 2010;185(11):6795-801.

43- Tangye SG, Ma CS, Brink R, Deenick EK. The good, the bad and the ugly - TFH cells in human health and disease. Nat Rev Immunol. 2013;13(6):412-26.

44- Teles RP, Gursky LC, Faveri M, Rosa EA, Teles FR, Feres $M$, et al. Relationships between subgingival microbiota and GCF biomarkers in generalized aggressive periodontitis. J Clin Periodontol. 2010;37(4):313-23.

45- Trombone AP, Claudino M, Colavite P, Assis GF, Avila-Campos MJ, Silva JS, et al. Periodontitis and arthritis interaction in mice involves a shared hyper-inflammatory genotype and functional immunological interferences. Genes Immun. 2010;11(6):479-89. 46- Trombone AP, Ferreira SB Jr, Raimundo FM, Moura KC, AvilaCampos MJ, Silva JS, et al. Experimental periodontitis in mice selected for maximal or minimal inflammatory reactions: increased inflammatory immune responsiveness drives increased alveolar bone loss without enhancing the control of periodontal infection. J Periodontal Res. 2009;44(4):443-51.

47- Van de Pavert SA, Mebius RE. New insights into the development of lymphoid tissues. Nat Ver Immunol. 2010;10(9):664-74.

48- Vernal R, Dezerega A, Dutzan N, Chaparro A, León R, Chandía $S$, et al. RANKL in human periapical granuloma: possible involvement in periapical bone destruction. Oral Dis. 2006;12(3):283-9.

49- Wan YY. Multi-tasking of helper T cells. Immunology. 2010;130(2):166-71.

50- Wright GP, Notley CA, Xue SA, Bendle GM, Holler A, Schumacher $\mathrm{TN}$, et al. Adoptive therapy with redirected primary regulatory $\mathrm{T}$ cells results in antigen-specific suppression of arthritis. Proc Natl Acad Sci USA. 2009;106(45):19078-83.

51- Zhang L, Li JM, Liu XG, Ma DX, Hu NW, Li YG, et al. Elevated Th22 cells correlated with Th17 cells in patients with rheumatoid arthritis. J Clin Immunol. 2011;31(4):606-14.

52- Zhu J, Paul WE. Heterogeneity and plasticity of T helper cells. Cell Res. $2010 ; 20(1): 4-12$. 